\title{
Regional Frontal Perfusion Deficits in Relapsing-Remitting Multiple Sclerosis with Cognitive Decline
}

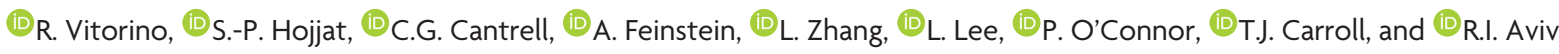

\begin{abstract}
BACKGROUND AND PURPOSE: Cortical dysfunction, quantifiable by cerebral perfusion techniques, is prevalent in patients with MS, contributing to cognitive impairment. We sought to localize perfusion distribution differences in patients with relapsing-remitting MS with and without cognitive impairment and healthy controls.
\end{abstract}

MATERIALS AND METHODS: Thirty-nine patients with relapsing-remitting MS (20 cognitively impaired, 19 nonimpaired) and 19 age- and sex-matched healthy controls underwent a neurocognitive battery and MR imaging. Voxel-based analysis compared regional deep and cortical GM perfusion and volume among the cohorts.

RESULTS: After we adjusted for localized volumetric differences in the right frontal, temporal, and occipital lobes, progressive CBF and CBV deficits were present in the left middle frontal cortex for all cohorts and in the left superior frontal gyrus for patients with cognitive impairment compared with patients without impairment and controls. Compared with healthy controls, reduced CBF was present in the limbic regions of patients with cognitive impairment, and reduced CBV was present in the right middle frontal gyrus in patients with cognitive impairment and in the temporal gyrus of relapsing-remitting MS patients without cognitive impairment.

CONCLUSIONS: Consistent regional frontal cortical perfusion deficits are present in patients with relapsing-remitting MS, with more widespread hypoperfusion in those with cognitive impairment, independent of structural differences, indicating that cortical perfusion may be a useful biomarker of cortical dysfunction and cognitive impairment in MS.

ABBREVIATIONS: BA $=$ Brodmann area; DARTEL $=$ Diffeomorphic Anatomical Registration Through Exponentiated Lie Algebra; $\mathrm{HC}=$ healthy controls; $\mathrm{MNI}=$ Montreal Neurological Institute; qCBF = quantitative cerebral blood flow; qCBV = quantitative cerebral blood volume; RRMS = relapsing-remitting multiple sclerosis; VBM $=$ voxel-based morphometry

$\mathrm{M}^{\mathrm{s}}$ $S$ is traditionally considered a demyelinating-inflammatory WM disorder; however, GM involvement is recognized in $50 \%-93 \%$ of patients, ${ }^{1,2}$ contributing to cognitive impairment, which is present in $40 \%-68 \%$ of cases. ${ }^{3,4}$ Patients with MS may display deficits in several cognitive domains, including working

Received January 6, 2016; accepted after revision March 17

From the Departments of Psychiatry (A.F.), Neurology (L.L.), and Medical Imaging (R.V., S.-P.H., L.Z., R.I.A.), Sunnybrook Health Sciences Centre, Toronto, Ontario, Canada; Departments of Medicine (L.L., P.O.), Psychiatry (A.F.), and Medical Imaging (S.-P.H., R.I.A.), University of Toronto, Toronto, Ontario, Canada; and Departments of Biomedical Engineering (C.G.C., T.J.C) and Radiology (T.J.C.), Northwestern University, Chicago, Illinois.

Dr. Aviv was supported by Canadian Institutes of Health Research operating grant (130366). Drs. Aviv and Hojjat are supported by a Biogen Fellowship Funding Award. Charles Cantrell is supported by the American Heart Association (14PRE20380310).

Dr. Carroll is supported by the US National Institutes of Health (1R21EB017928-01A1)

Please address correspondence to Rita Vitorino, BSc, Sunnybrook Health Sciences Centre, 2075 Bayview Ave, Room AB204, Toronto, Ontario, Canada M4N 3M5; e-mail: rita.vitorino@sunnybrook.ca

- Indicates open access to non-subscribers at www.ajnr.org

http://dx.doi.org/10.3174/ajnr.A4824 memory, learning and memory retrieval, executive function, and especially information-processing speed., ${ }^{2,5}$

Multiple studies have quantified the relative contributions of WM T2 hyperintense lesions and, to a lesser extent, GM cortical lesions to cognition in MS. The relationship between WM T2 hyperintense lesion burden and cognitive impairment is modest, ${ }^{6}$ and GM and WM damage may occur interdependently, ${ }^{1}$ with cortical abnormalities reported in the absence of WM disease. ${ }^{7}$ Both atrophy and cortical lesion load are important predictors of cognitive deficits in patients with $\mathrm{MS}^{5}$; nevertheless, cortical lesion burden is increasingly reported as a stronger and independent predictor of cognitive performance in comparison with cortical volume. ${ }^{8}$

Current clinical imaging techniques used for cortical lesion detection, such as double inversion recovery, detect few lesions (around 18\%) compared with histopathologic studies. ${ }^{9}$ Several studies have proposed new strategies to detect cortical abnormalities, including cortical lesion volume or more subtle ultrastructural (magnetization transfer ratio ${ }^{10,11}$ and DTI ${ }^{12,13}$ ) or perfusion 
abnormalities, in the clinical setting. GM is inherently sensitive to perfusion changes caused by both physiologic and pathologic alterations, due to its high vascularity and metabolic activity. Cortical perfusion can be evaluated with multiple imaging techniques, including fMRI, arterial spin-labeling, and gadoliniumbased MR imaging techniques, such as DSC, which is the most widely performed clinical perfusion technique. By using pre- and postgadolinium scans to calibrate DSC, the bookend technique offers accurate cerebral perfusion quantification with high PET correlation and interobserver reliability. ${ }^{14,15}$

Previous perfusion studies have shown that regardless of MS clinical subtype, cerebral hypoperfusion is an early and integral occurrence, ${ }^{16,17}$ including in early relapsing-remitting MS (RRMS), in which reduction may be seen in the absence of structural differences compared with healthy controls (HC). ${ }^{18}$ Studies explicitly exploring cognitive impairment in both RRMS and secondary-progressive MS describe significant and focal frontal cortical correlations between $\mathrm{CBV}$ and $\mathrm{CBF}$ reductions and cognitive deficits. ${ }^{16,19-21}$

In the present study, we sought to localize CBF and CBV in $\mathrm{HC}$ and RRMS patients with and without cognitive impairment to determine whether a similar pattern of involvement is present compared with that previously reported for secondary-progressive multiple sclerosis. We hypothesized that patients with RRMS with cognitive impairment similarly exhibit localized frontal cerebral $\mathrm{CBF}$ and $\mathrm{CBV}$ reduction in functionally consistent brain regions, compared with patients without impairment and $\mathrm{HC}$. We further evaluated the consistency of the localized findings before and after accounting for any structural group differences.

\section{MATERIALS AND METHODS Patient Cohort}

Thirty-nine patients with RRMS (modified McDonald criteria, $2010^{22}$ ) were prospectively recruited during a 1-year period from 2 tertiary referral MS clinics at Sunnybrook and St. Michael's hospitals. Initially, 20 patients with cognitive impairment were recruited followed by the remaining patients without impairment and $19 \mathrm{HC}$ (with no previous history of neurologic disorders) who were selected to reflect the overall distribution of sex and age of the impaired cohort. Charts of potential patients were reviewed by a senior neurologist (20 years' experience) before recruitment. Exclusion criteria included relapse or corticosteroid use within the past 3 months; history of drug/alcohol abuse; premorbid (preMS) psychiatric history; head injury, including loss of consciousness; concurrent morbidity; and MR imaging/gadolinium contraindications. All study participants were purposely recruited for this study. At the time of consent, the small potential risks associated with gadolinium injection were discussed, referencing American College of Radiology and FDA communications. Consent was obtained following confirmation of MR imaging (and gadolinium) eligibility on the basis of a standardized MR imaging contraindication questionnaire and glomerular filtration rate determination. The study was approved by the research ethics board of both Sunnybrook Health Sciences Centre and St. Michael's Hospital, and informed consent was obtained from all participants.

\section{Neurocognitive Testing}

All patients underwent clinical assessments within 1 week of image acquisition, documenting demographic data and medical history, including relapse history. Disability was assessed by using the Expanded Disability Status Scale. ${ }^{23}$ All participants were tested by using the Minimal Assessment of Cognitive Function in Multiple Sclerosis battery covering 5 cognitive domains: working memory and processing speed (Paced Auditory Serial Addition Test; Symbol Digit Modalities Test); learning and memory (Brief Visual Memory Test-Revised; California Verbal Learning TestII); executive function (Delis-Kaplan Executive Function System); verbal fluency (Controlled Word Association Test); and visuospatial perception (Judgment of Line Orientations). ${ }^{24}$ Raw scores of each individual test were converted to $z$ scores by using widely available normative data, which correct for age and sex. Norms for 3 of the key components of the neurocognitive battery (Paced Auditory Serial Addition Test; Symbol Digit Modalities Test; Controlled Word Association Test) also correct for education. Patients scoring 1.5 SDs below normative data on $\geq 2$ cognitive tests were considered cognitively impaired. ${ }^{25}$ The Hospital Anxiety and Depression Scale was also administered.

\section{MR Imaging Acquisition}

All scans were acquired on a $3 \mathrm{~T}$ MR imaging system (Achieva; Philips Healthcare, Best, the Netherlands) with an 8-channel phased array head coil receiver. Conventional MR imaging sequences were acquired for structural and lesion characterization, including axial volumetric TSE T1 (TR/TE/flip angle $=9.5 / 2.3$ $\mathrm{ms} / 12^{\circ} ; \mathrm{FOV}=24 \mathrm{~cm}$; acquisition matrix $=256 \times 219$; section thickness $=1.2 \mathrm{~mm})$; axial proton-density/T2 (TR/TE/flip angle $=2500 / 10.7 \mathrm{~ms} / 90^{\circ}$; FOV $=23 \mathrm{~cm}$; acquisition matrix $=$ $256 \times 263$; section thickness $=3 \mathrm{~mm}$ ); axial phase-sensitive inversion recovery $(\mathrm{TR} / \mathrm{TE}=3374 / 15 \mathrm{~ms} ; \mathrm{FOV}=23 \mathrm{~cm}$; acquisition matrix $=400 \times 255$; section thickness $=3 \mathrm{~mm})$; and axial field-echo echo-planar DSC (TR/TE/flip angle $=1633 / 30 \mathrm{~ms} / 60^{\circ}$; FOV $=22 \mathrm{~cm}$; acquisition matrix $=96 \times 93$; section thickness $=$ $4 \mathrm{~mm}$; no gap; signal bandwidth $=1260 \mathrm{~Hz} /$ pixel; sections $=24$ ). A segmented inversion recovery Look-Locker EPI sequence was performed immediately before and after the axial DSC sequence $\left(\mathrm{TR} / \mathrm{TE} /\right.$ flip angle $=29 / 14 \mathrm{~ms} / 20^{\circ} ; \mathrm{TI}=15.8 \mathrm{~ms} ; \mathrm{FOV}=22 \mathrm{~cm}$; acquisition matrix $=128 \times 126 ; 15$ lines in $k$-space per acquisition; section thickness $=4 \mathrm{~mm}$; 60 time points). Ten milliliters of $1 \mathrm{mmol} / \mathrm{mL}$ concentration of gadobutrol (Gadovist; Bayer Schering Pharma, Berlin, Germany) was administered by power injector at a rate of $5 \mathrm{~mL} / \mathrm{s}$, followed by a $25-\mathrm{mL}$ bolus of saline at 5 $\mathrm{mL} / \mathrm{s}$. Sixty images were acquired at 1.6-second intervals with the injection occurring at the fifth volume. A 3-second delay was placed after the last imaging time point to facilitate longitudinal magnetization recovery.

\section{Image Processing}

Perfusion Maps. Quantitative CBF (qCBF) and CBV (qCBV) maps were generated from the DSC and Look-Locker echo-planar images (T1-weighted pre- and postgadolinium reference scans) by using the bookend technique. ${ }^{15}$ Briefly, these Look-Locker EPI scans allow DSC calibration, independent of an arterial input function, by quantifying WM T1 signal changes relative to the 
blood pool to calculate the steady-state CBV in WM by using a water-correction factor to correct for intra- to extravascular water exchange. Deconvolution of tissue-concentration-time curves by the arterial input function by using singular value decomposition yields the relative $\mathrm{CBF}$, while relative $\mathrm{CBV}$ is determined by calculating the ratio of the area under the tissue-concentration-time curve and the arterial input function. Final perfusion quantification of $\mathrm{qCBV}$ and $\mathrm{qCBF}$ is then performed as previously described. ${ }^{26}$

Lesion Load. Structural T1- and proton-density/T2-weighted images were coregistered by using linear registration (SPM8 software; http://www.fil.ion.ucl.ac.uk/spm/). Lesions were manually traced with Analyze 8.0 software (AnalyzeDirect, Overland Park, Kansas) by an experienced clinician (10 years' experience) by using phase-sensitive inversion recovery for cortical lesion tracing and proton-density/T2 and T1 scans for WM T2 hyperintense lesions and T1 black hole tracing, respectively.

Voxel-Based Morphometry Analysis. Voxel-based morphometry (VBM) analysis was performed in SPM8 by using Diffeomorphic Anatomical Registration Through Exponentiated Lie Algebra (DARTEL) and the unified segmentation model for structural and perfusion images, respectively. ${ }^{27,28}$

Structural VBM. T1 structural images were segmented by using both a unified segmentation model and DARTEL functions in SPM8 and then checked for accuracy. A group-specific template was created by using the DARTEL space segmentations. Each participant's native space segmentations were registered to this template with a nonlinear transformation; then they were affinetransformed into Montreal Neurological Institute (MNI, McGill University) space before being smoothed with an 8-mm full width at half maximum isotropic Gaussian kernel. The segmentations were aligned to MNI152 space via the DARTEL template by using the same transformations in a single step.

Perfusion VBM. A mean DSC series was constructed for each patient by averaging the 60 EPI DSC acquisitions and then normalizing them to MNI152 space by using SPM8. A group-specific perfusion template was then created in MNI space. The DSC sequence was linearly registered to the group template by using the FMRIB Linear Image Registration Tool (FLIRT; http://www. fmrib.ox.ac.uk) followed by nonlinear intensity modulation and multiresolution nonlinear registration with 4 subsampling levels (FMRIB Nonlinear Registration Tool, FNIRT; http://fsl. fmrib.ox.ac.uk/fsl/fslwiki/FNIRT). ${ }^{29}$ These sequences were smoothed at each respective resolution level during the registration by using full width at half maximum Gaussian kernels of 6, 4, 2, and $2 \mathrm{~mm}$. This transformation matrix was then applied to the intrinsically coregistered bookend perfusion maps of $\mathrm{qCBF}$ and $\mathrm{qCBV}$.

\section{Statistical Analysis}

Clinical and Demographic Measures. Demographic, neurologic, and neuropsychological data were summarized in $\mathrm{HC}$ and patients with RRMS with and without cognitive impairment by using means and SDs for continuous variables and counts for categoric variables. Statistical Analysis Software (SAS, Version 9.4; SAS Institute, Cary, North Carolina) was used to compare each clinical, demographic, and volumetric measure among the 3 groups; general linear regression or logistic regression analysis was applied for continuous or categoric variables. Any variables demonstrating significant group differences $(P<.017, P<.05$ corrected for multiple comparisons among the 3 cohorts) were included as covariates for the respective mass univariate analysis.

VBM Analysis. Perfusion maps and structural images were compared by using the mass univariate technique used by SPM. On the basis of previous research, ${ }^{16,19}$ we hypothesized cortical perfusion changes in the frontal cortex. With this a priori hypothesis, VBM analysis was restricted to GM and clusters with $\geq 20$ contiguous voxels, with a voxelwise $P$ value threshold of $P<.001$ considered significant. VBM analysis was repeated for perfusion measures with structural findings as covariates. Brain regions identified by SPM as statistically significant were identified by using xjView software 8.12 (http://www.alivelearn.net/xjview).

\section{RESULTS}

\section{Demographic, Clinical, and Volumetric Data}

Demographic, clinical, and volumetric data are summarized in Table 1. Similar group characteristics were present with the exception of lower education in patients with RRMS with cognitive impairment compared with $\mathrm{HC}(P=.004)$. RRMS patients with and without cognitive impairment scored higher on the anxiety measure than $\mathrm{HC}(P=.0004$ and $P=.012$, respectively), and patients with cognitive impairment also showed higher depression scores compared with those with RRMS without impairment and HC $(P<.0001, P=.0001)$. Furthermore, patients with cognitive impairment were more functionally disabled compared with those without impairment $(P=.014)$ as measured by the Expanded Disability Status Scale. With respect to structural/volumetric differences, patients with RRMS with cognitive impairment had a reduction in WM $(P=.008)$ and thalamic volume $(P=.014)$.

\section{Neurocognitive Performance}

There was no difference in cognitive performance between HC and those with nonimpaired RRMS. Patients with RRMS with cognitive impairment performed significantly worse on all cognitive tests compared with both $\mathrm{HC}$ and those without impairment (Table 1), except for the Delis-Kaplan Executive Function System and the Judgment of Line Orientations test.

\section{VBM Data (Perfusion and Structural)}

Mass univariate SPM analysis detected significantly reduced $\mathrm{qCBF}$ and $\mathrm{qCBV}$ in the left middle frontal gyrus (encompassing Brodmann areas [BAs] 10,11,46) for all group comparisons $\left(P_{\text {uncorrected }}<.001\right)$. Patients with cognitive impairment showed qCBF and qCBV reduction compared with those with RRMS without impairment and $\mathrm{HC}$ in the bilateral superior frontal gyrus (BAs 6, 8, 10), left fusiform gyrus (BA 20), and right limbic lobe, including the cingulate gyrus (BA 24).

Compared with unimpaired RRMS, those with RRMS and cognitive impairment showed lower qCBF in the left thalamus (including the medial dorsal nuclei) and lower qCBV in the right anterior cingulate (BA 25), left posterior cingulate (BA 31), right inferior parietal lobule (BA 40), right lingual gyrus, and left cau- 
Table 1: Demographic, neurologic, and neuropsychological data of healthy controls and patients with RRMS

\begin{tabular}{|c|c|c|c|}
\hline & $\begin{array}{l}\text { Healthy Controls } \\
\qquad(n=19)\end{array}$ & $\begin{array}{c}\text { Cognitively } \\
\text { Nonimpaired } \\
\text { RRMS }(n=19)\end{array}$ & $\begin{array}{c}\text { Cognitively } \\
\text { Impaired RRMS } \\
(n=20)\end{array}$ \\
\hline \multicolumn{4}{|l|}{ Demographic and clinical data } \\
\hline Age (yr) & $49.0 \pm 7.1$ & $46.4 \pm 7.2$ & $48.1 \pm 4.7$ \\
\hline $\operatorname{Sex}(F / M)$ & 14:5 & $15: 4$ & $12: 8$ \\
\hline Education (yr) & $16.9 \pm 2.9^{b}$ & $16.1 \pm 1.3$ & $14.6 \pm 1.9^{b}$ \\
\hline Disease duration (yr) & NA & $11.8 \pm 5.4$ & $11.6 \pm 4.9$ \\
\hline EDSS & NA & $1.8 \pm 0.7^{c}$ & $2.6 \pm 0.7^{c}$ \\
\hline HADS-Anxiety & $4.4 \pm 4.3^{\mathrm{b}, \mathrm{d}}$ & $6.37 \pm 3.1^{d}$ & $8.5 \pm 3.7^{b}$ \\
\hline HADS-Depression & $2.3 \pm 2.3^{b}$ & $3.5 \pm 3.2^{c}$ & $7.6 \pm 2.9^{b, c}$ \\
\hline Treatment & NA & & \\
\hline$\beta$-interferon & & $4(21 \%)$ & $3(15 \%)$ \\
\hline Other immune suppressors & & $11(58 \%)$ & $12(60 \%)$ \\
\hline None & & $4(21 \%)$ & $5(25 \%)$ \\
\hline $\begin{array}{l}\text { Presence of enhancing lesions } \\
\text { Volumetric data }\left(\mathrm{cm}^{3}\right)\end{array}$ & NA & $1(5 \%)$ & $5(25 \%)$ \\
\hline GM & $653.37 \pm 81.51$ & $618.83 \pm 53.94$ & $605.09 \pm 60.90$ \\
\hline WM & $458.22 \pm 65.02^{b}$ & $421.84 \pm 39.29$ & $414.53 \pm 71.56^{b}$ \\
\hline BG & $19.41 \pm 2.75$ & $18.68 \pm 2.52$ & $18.04 \pm 2.93$ \\
\hline Th & $9.83 \pm 1.92^{b}$ & $9.14 \pm 1.98$ & $7.91 \pm 1.88^{b}$ \\
\hline $\mathrm{CL}$ & $0.00 \pm 0.00^{b}$ & $0.12 \pm 0.11$ & $0.22 \pm 0.36^{b}$ \\
\hline $\mathrm{T} 2 \mathrm{H}$ & $0.00 \pm 0.00^{b}$ & $9.37 \pm 10.02$ & $13.47 \pm 13.30^{\mathrm{b}}$ \\
\hline Tlbh & $0.00 \pm 0.00^{b}$ & $3.21 \pm 2.98$ & $5.85 \pm 6.77^{b}$ \\
\hline CSF & $320.89 \pm 210.43^{b}$ & $353.22 \pm 131.71$ & $400.29 \pm 173.78^{b}$ \\
\hline \multicolumn{4}{|l|}{ Neurocognitive tests (z score) } \\
\hline COWAT-FAS & $-0.67 \pm 0.83$ & $-0.26 \pm 1.06^{c}$ & $-1.16 \pm 0.89^{c}$ \\
\hline COWAT-Animals & $-0.13 \pm 1.14$ & $-0.41 \pm 0.95^{c}$ & $-0.59 \pm 1.18^{c}$ \\
\hline BVMT-IR & $-0.37 \pm 1.15^{b}$ & $-0.07 \pm 1.04^{c}$ & $-1.68 \pm 1.34^{\mathrm{b}, \mathrm{c}}$ \\
\hline BVMT-DR & $-0.40 \pm 1.14^{b}$ & $-0.42 \pm 0.77^{c}$ & $-1.62 \pm 1.48^{b, c}$ \\
\hline PASAT-3 & $-0.39 \pm 0.94^{b}$ & $-0.05 \pm 0.61^{c}$ & $-1.71 \pm 0.82^{b, c}$ \\
\hline PASAT-2 & $-0.21 \pm 0.89^{b}$ & $-0.26 \pm 0.66^{c}$ & $-1.80 \pm 0.57^{\mathrm{b}, \mathrm{c}}$ \\
\hline JLO & $-0.98 \pm 0.20$ & $-0.83 \pm 0.56$ & $-0.40 \pm 0.67$ \\
\hline SDMT & $-0.14 \pm 0.92^{b}$ & $-0.02 \pm 0.75^{c}$ & $-1.80 \pm 1.17^{\mathrm{b}, \mathrm{c}}$ \\
\hline CVLT II-IR & $-0.25 \pm 1.05^{\mathrm{b}}$ & $-0.23 \pm 1.04^{c}$ & $-1.94 \pm 1.36^{\mathrm{b}, \mathrm{c}}$ \\
\hline CVLT II-DR & $-0.11 \pm 0.66^{b}$ & $-0.21 \pm 0.92^{c}$ & $-2.20 \pm 1.61^{b, c}$ \\
\hline DKEFS-ST & $-0.51 \pm 0.73$ & $-0.26 \pm 0.61$ & $-0.20 \pm 1.25$ \\
\hline
\end{tabular}

Note:-NA indicates not applicable; EDSS, Expanded Disability Status Score; HADS, Hospital Anxiety and Depression Scale; COWAT, Controlled Oral Word Association Test; BVMT, Brief Visuospatial Test- Revised; PASAT, Paced Auditory Serial Addition Test; JLO, Judgment of Line Orientation Test; SDMT, Symbol Digit Modalities Test; CVLT II, California Verbal Learning Test-II; IR, immediate recall; DR, delayed recall; DKEFS-ST, Delis-Kaplan Executive Function System Sorting Test; BG, basal ganglia; Th, thalamus; $\mathrm{CL}$, cortical lesions; $\mathrm{T} 2 \mathrm{H}, \mathrm{T} 2$ hyperintensities; T1bh, $\mathrm{T} 1$ black holes.

a Significance at $P<.017$, corrected for multiple comparisons; all values are means unless specified.

${ }^{\mathrm{b}}$ Healthy controls vs patients with RRMS with cognitive impairment.

c Patients with RRMS without impairment vs those with cognitive impairment

${ }^{\mathrm{d}}$ Healthy controls vs patients with RRMS without impairment.

date. Furthermore, those with RRMS and cognitive impairment showed qCBF reductions compared with $\mathrm{HC}$ in the right middle frontal gyrus (BA 6, 10) and qCBV deficits in the right precentral (BA 4) and right parahippocampal gyri (BA 28).

Regional volume of the right superior frontal gyrus (BA 6, 10) was decreased in those with cognitively impaired RRMS compared with those without it, and those without impairment compared with healthy controls. Additionally, patients with RRMS and cognitive impairment showed focal atrophy in the right precentral (BA 6) and transtemporal gyri (BA 42) compared with patients with nonimpaired RRMS and in the right inferior occipital gyrus (BA 18) compared with HC.

VBM analysis conducted with regional volumes of focal atrophy included as covariates found that cortical hypoperfusion ( $\mathrm{qCBF}$ and $\mathrm{qCBV}$ ) was maintained in the left middle frontal gyrus (BAs 10, 11, 46) for all group comparisons and in the left superior frontal gyrus (BAs 6, 10) for patients with RRMS and cognitive impairment compared with both patients without impairment and $\mathrm{HC}$ (Figure and Table 2). Patients with cognitive impairment continued showing qCBV deficits in the right lingual gyrus (with additional qCBF reduction in the left BA 18), right inferior parietal lobule (BA 40), and left fusiform gyrus (BA 20) and $\mathrm{qCBF}$ reductions in the caudate head and thalamic medial dorsal nuclei in comparison with those with RRMS without impairment, and decreased qCBF in the right middle frontal gyrus (BA 6) and decreased qCBV in the left parahippocampal gyrus (BA 28) in comparison with HC. Reduced qCBV in cognitively impaired compared with nonimpaired patients with RRMS was present in the left inferior frontal gyrus (BA 46) and diminished qCBF, in the right caudate body. Compared with $\mathrm{HC}$, patients without impairment showed reduced $\mathrm{qCBF}$ in the superior temporal lobe (BA 38).

\section{DISCUSSION}

Consistent perfusion deficits in the frontal cortex are present in patients with RRMS independent of global or regional atrophy. Significantly different and progressive $\mathrm{qCBF}$ and $\mathrm{qCBV}$ reduction among all groups was demonstrated in the middle frontal cortex and the left superior frontal gyrus in the impaired RRMS compared with the other 2 cohorts, after considering confounding variables of disability, anxiety, depression, and education. Patients with RRMS and HC were further distinguished by qCBV reductions in the right limbic and qCBF reductions in the right frontal (for impaired) and right temporal region (for nonimpaired). Finally, qCBV deficits were found in cognitively impaired compared with nonimpaired patients with RRMS in the left frontal (inferior frontal gyrus), right parietal (inferior parietal lobule), left temporal (fusiform gyrus), and bilateral occipital (lingual gyrus) lobes, and qCBF deficits, in deep GM structures, including the bilateral caudate and the left thalamus (medial dorsal nuclei).

Distribution of $\mathrm{qCBF}$ and $\mathrm{qCBV}$ reductions in the superior frontal, middle frontal, and parahippocampal gyri is similar to that reported in a recent pseudocontinuous arterial spin-labeling study comparing HC and patients with very early RRMS. ${ }^{18}$ That study also showed additional qCBF reduction in multiple other areas not demonstrated in the present study; however, the discrepancies could be explained by different MR imaging techniques (pseudocontinuous arterial spin-labeling versus bookend perfusion) and patient populations. Unlike findings in our RRMS 


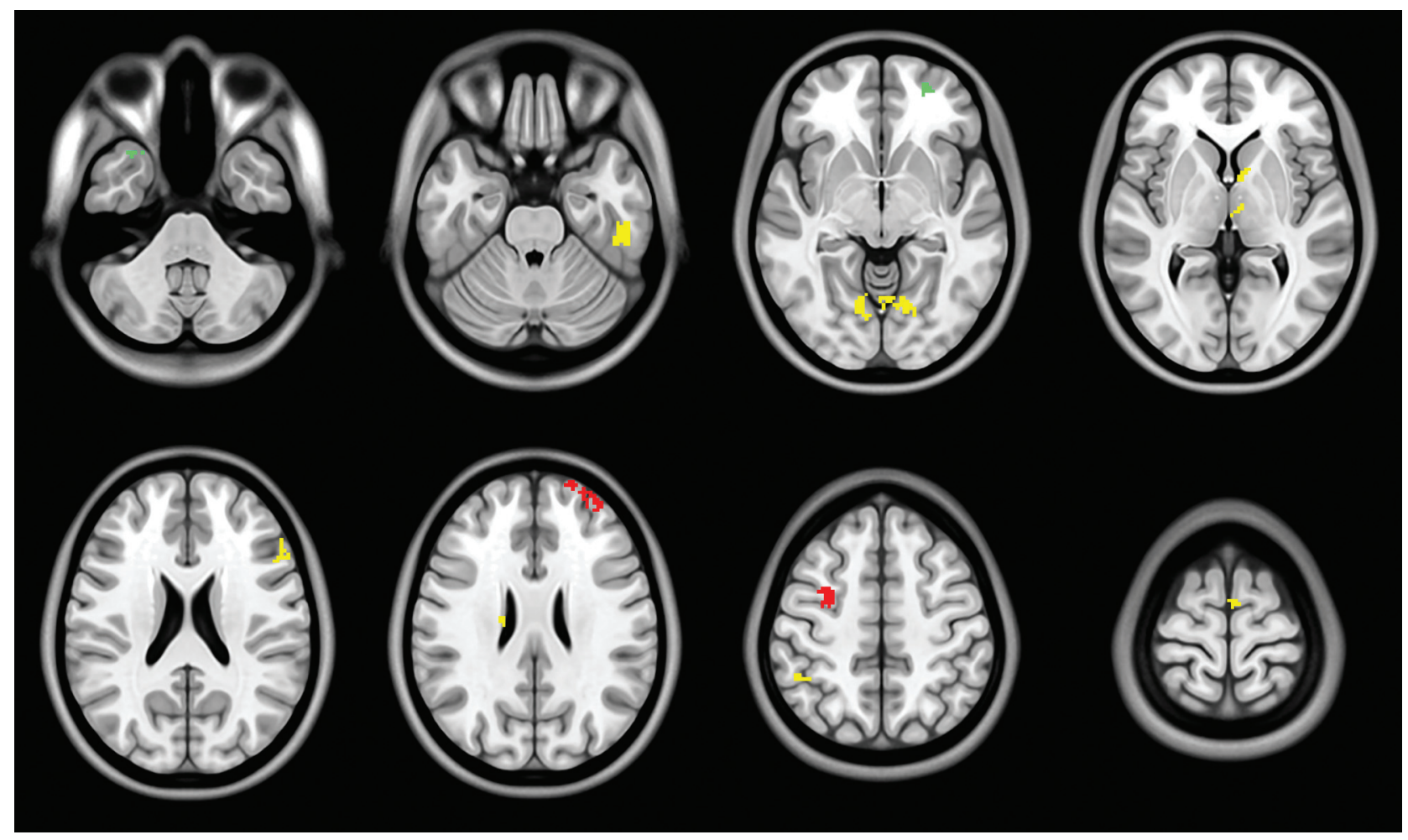

FIGURE. Areas of significantly $\left(P_{\text {uncorrected }}<.001\right)$ reduced cortical perfusion in RRMS subgroups and healthy controls, with volumes for atrophied regions added as covariates. Green indicates healthy controls versus nonimpaired RRMS; red, healthy controls versus cognitively impaired RRMS; and yellow, nonimpaired RRMS versus cognitively impaired RRMS.

Table 2: Areas of significantly $\left(P_{\text {uncorrected }}<.001\right)$ reduced cortical perfusion in RRMS subgroups and healthy controls, with volumes for atrophied regions added as covariates

\begin{tabular}{|c|c|c|c|c|c|c|c|c|c|}
\hline \multirow[b]{2}{*}{ qCBF } & \multirow[b]{2}{*}{ qCBV } & \multirow[b]{2}{*}{ Anatomic Regions } & \multirow[b]{2}{*}{$\begin{array}{l}\text { Cluster } \\
\text { Size }\end{array}$} & \multicolumn{3}{|c|}{ MNI Coordinates } & \multicolumn{3}{|c|}{$t$ Values } \\
\hline & & & & $\mathbf{x}$ & $y$ & $z$ & $\begin{array}{l}\mathrm{HC} \\
\text { vs Cl }\end{array}$ & $\begin{array}{c}\text { HC } \\
\text { vs NI }\end{array}$ & $\begin{array}{c}\mathrm{NI} \\
\text { vs Cl }\end{array}$ \\
\hline \multirow{5}{*}{$\begin{array}{l}\sqrt{ } \\
\sqrt{ } \\
\sqrt{ }\end{array}$} & \multirow[t]{2}{*}{$\sqrt{ }$} & Left superior frontal gyrus (BAs 6, 10) & 78 & -32 & 50 & 28 & 3.52 & \multirow{4}{*}{4.56} & \multirow[t]{2}{*}{3.62} \\
\hline & & Right middle frontal gyrus (BA 6) ${ }^{\mathrm{a}}$ & 26 & 34 & 0 & 64 & 3.31 & & \\
\hline & \multirow[t]{2}{*}{$\sqrt{ }$} & Left middle frontal gyrus $(B A s 10,11,46)^{a}$ & 100 & -22 & 56 & 26 & 4.79 & & 3.21 \\
\hline & & Left inferior frontal gyrus (BA 46) & 21 & -48 & 30 & 20 & & & 3.29 \\
\hline & $\sqrt{ }$ & Right parahippocampal gyrus (BA 28) & 27 & 24 & -22 & -12 & 4.15 & & \\
\hline \multirow{3}{*}{$\begin{array}{l}\sqrt{ } \\
\sqrt{ }\end{array}$} & \multirow[t]{2}{*}{$\sqrt{ }$} & Right lingual gyrus ${ }^{\mathrm{a}}$ & 101 & 12 & -72 & -2 & & & 5.11 \\
\hline & & Left lingual gyrus (BA 18) & 72 & -6 & -68 & 2 & & & 3.99 \\
\hline & \multirow[t]{2}{*}{$\checkmark$} & Right inferior parietal lobule (BA 40) ${ }^{\mathrm{a}}$ & 22 & 48 & -40 & 56 & & & 3.98 \\
\hline \multirow[t]{2}{*}{$\sqrt{ }$} & & Right superior temporal gyrus (BA 38) & 38 & 28 & 10 & -46 & & 3.64 & \\
\hline & \multirow[t]{4}{*}{$\checkmark$} & Left temporal fusiform gyrus (BA 20) & 134 & -44 & -22 & -30 & & & 3.74 \\
\hline$\checkmark$ & & Left caudate head ${ }^{\mathrm{a}}$ & 36 & -10 & 6 & 4 & & & 3.64 \\
\hline$\sqrt{ }$ & & Right caudate body & 24 & 18 & -20 & 26 & & & 3.68 \\
\hline$\sqrt{ }$ & & Left thalamic medial dorsal nuclei ${ }^{a}$ & 31 & -6 & -18 & 6 & & & 3.63 \\
\hline
\end{tabular}

Note:- Cl indicates patients with RRMS with cognitive impairment; NI, patients with RRMS without impairment; $\sqrt{ }$, anatomic region was present in VBM analysis for this map.

${ }^{a}$ Anatomic regions remained significant from previous VBM analysis without atrophy areas added as covariates.

patients without impairment, who are cognitively indistinguishable from healthy controls, Debernard et $\mathrm{al}^{18}$ reported a borderline significant Brief Visuospatial Test reduction and demonstrated a lower white matter volume in their early RRMS cohort, suggesting a greater level of disease burden in the patient sample (supported by a higher upper Expanded Disability Status Scale score of 4.5 compared with 3.5 in our sample). In contrast to that study, we demonstrated regional cortical GM volume reduction within the right frontal, temporal, and occipital lobes consistent with that observed by Riccitelli et al. ${ }^{30}$ Reduced superior frontal gyrus, thalamic, and caudate nuclei perfusion was similarly re- ported in a secondary-progressive multiple sclerosis with cognitive impairment patient cohort, suggesting that the frontal reduction may be a marker of impairment in patients with both RRMS and secondary-progressive multiple sclerosis, even after controlling for structural differences. ${ }^{19}$

The frontal areas, BAs 6, 10, and 46, affected in our patients with RRMS, are responsible for memory processing, particularly working memory, memory encoding, and retrieval. ${ }^{31,32}$ Several studies relate BA 10 with prospective memory and "intentional forgetting," suggesting involvement of BA 10 in controlling and manipulating memory. ${ }^{32,33}$ BA 46 activation is associated with 
working memory processes and memory manipulation. ${ }^{31,33}$ It has been assumed that working memory is involved in a diversity of cognitive processes, including planning, ${ }^{34}$ reasoning, ${ }^{35}$ and problem-solving. ${ }^{36}$ On the other hand, involvement of BA 6 in memory and attention may be due to the activation of an extended brain network in which the middle frontal gyrus has a fundamental task in memory strategy organization and memory control. ${ }^{37}$ Hypoperfusion (qCBF and qCBV) in the left middle frontal and right superior temporal gyri with preservation of perfusion within the remaining medial prefrontal cortex in patients with RRMS who are nonimpaired compared with those who are cognitively impaired likely reflects increased cortical plasticity, because the medial prefrontal cortex has been previously shown to adaptively compensate for functional impairment in patients with $\mathrm{MS} .{ }^{38} \mathrm{~Pa}-$ tients with RRMS and secondary-progressive multiple sclerosis performing a processing speed and attention task (Counting Stroop Task) were found to have activation predominantly in the left medial frontal region (left middle frontal gyrus/superior frontal sulcus and bilateral superior frontal gyri, corresponding to BAs $8,9,10$ ), while $\mathrm{HC}$ had greater right frontal activation (inferior frontal gyrus, BA 45; and right basal ganglia). ${ }^{38}$

Last, BAs 28 and 38 are also implicated in memory, particularly nonverbal memory (right parahippocampal gyrus) ${ }^{39}$ and multimodal memory retrieval (superior temporal gyrus). ${ }^{40}$ Supporting the validity of the structural and progressive perfusion differences in patients with RRMS with cognitive impairment described above, significant impairment in working memory (Paced Auditory Serial Addition Test and Symbol Digit Modalities Test), visual and verbal learning, and memory retrieval (Brief Visual Memory Test-Revised and California Verbal Learning Test-II) was present compared with patients who were nonimpaired and $\mathrm{HC}$.

Our VBM analysis by necessity controlled for a number of important potential confounding covariates; for example, the effect of depression was accounted for by the inclusion of Hosptial Anxiety and Depression Scale. Differing educational levels among cohorts was accounted for by the "normalization" of raw neurocognitive battery scores against representative population datasets. Cortical lesions and, to a lesser extent, T2 hyperintense lesion burden are both implicated in cognitive impairment of patients with MS. Comparisons between patient groups and HC included lesion volumes as covariates in our VBM analysis. However, no significant difference in lesion burden was present between MS groups, precluding a quid pro quo comparison.

Cerebral blood volume (amount of blood in $100 \mathrm{~g}$ of brain tissue) and blood flow (amount of blood flowing through $100 \mathrm{~g}$ of brain tissue per minute) abnormalities are found in a number of neurologic conditions such as stroke, characterized by ischemia. The physiopathology leading to cerebral hypoperfusion is unknown and may be multifactorial. While evidence does not support a primary neuronal loss mechanism given multiple findings of reduced cortical perfusion in the absence of GM volume loss, ${ }^{16,18,19}$ mitochondrial disturbances and vascular abnormalities have been implicated in cerebral hypoperfusion in MS. Mitochondrial dysfunction can contribute to cerebral hypoperfusion in the form of a diminished mitochondrial capacity resulting from reductions in gene products specific for the mitochondrial elec- tron transport chain ${ }^{41}$ or due to intra-axonal mitochondrial pathology triggered by macrophage-derived reactive oxygen and nitrogen species, which may precede axonal damage. ${ }^{42}$ Cerebral hypoperfusion can also be secondary to vascular abnormalities. Increased levels of endothelin-1, a potent vasoconstrictive peptide, are found in patients with MS, suggesting that cerebral blood flow reductions are mediated by elevated levels of this peptide. ${ }^{43}$ Astrocytes of patients with MS are deficient in the $\beta 2$-adrenergic receptor, resulting in cellular metabolic dysfunction affecting potassium uptake after synaptic activity and its subsequent release to the perivascular space, thus reducing arteriolar vasodilation. ${ }^{44}$

Venous changes are also well-described in MS; and given that venous capacitance accounts for approximately $70 \%$ of CBV, pathologies that decrease venous capacitance should greatly impact qCBV. For example, Ge et $\mathrm{al}^{45}$ demonstrated reduced visibility of periventricular venous vasculature in patients with MS by using susceptibility-weighted imaging. The authors suggested that this reduction could be attributable to decreased vein number or size secondary to venous occlusion and perivenular inflammation. Such pathology could also be driven by obliterative vasculitis, which preferentially disrupts venous changes. ${ }^{46,47}$ Additionally, intrastriatal injections of proinflammatory cytokine tumor necrosis factor- $\alpha$, found elevated in MS brains, ${ }^{48}$ in rat models resulted in significant reductions of cerebral blood flow. ${ }^{49}$ Cerebral hypoperfusion is characterized by both blood flow and volume changes, and these perfusion metrics may be differentially affected by the physiopathologic methods proposed. Additional studies should be conducted to explore the differences in cerebral blood flow and volume and their relation to physiopathology.

Limitations include the need for contrast agent injection required for DSC perfusion, precluding its use in patients with contraindications such as renal impairment. DSC is a relatively lowresolution technique in comparison with structural imaging but comparable with other functional techniques such as diffusion tensor and arterial spin-labeling techniques, which were previously applied to MS. DSC enables whole-brain scanning in approximately 2 minutes, therefore, minimally prolonging scanning time with higher signal-to-noise than arterial spin-labeling. Because the classes of disease-modifying drugs were evenly represented in both cognitively impaired and nonimpaired groups, we did not adjust for this factor. However, given that the effects of such treatments on cortical perfusion abnormalities are unclear, it would be prudent to adjust for disease-modifying drugs in future studies if difference occurs. Similarly, fatigue, experienced by $78 \%-90 \%$ of patients with MS, ${ }^{50}$ may be associated with impaired cognitive function and should be accounted for in future studies. ${ }^{51}$ Despite the relatively small sample size, consistent frontal perfusion deficits were demonstrated in our RRMS sample. According to our a priori hypothesis, this comparison was uncorrected but included several confounders. These results should be validated in a larger patient cohort. Longitudinal studies would also be helpful in determining whether perfusion measurements are sensitive to disease progression.

\section{CONCLUSIONS}

Consistent regional frontal cortical perfusion deficits are found in patients with RRMS, with more widespread hypoperfusion in 
cognitively impaired RRMS, independent of structural differences. Our findings suggest a potential role for cortical perfusion as a useful biomarker of cortical dysfunction and cognitive impairment in MS.

Disclosures: Charles G. Cantrell—RELATED: Grant: American Heart Association (14PRE20380810).* Liesly Lee-UNRELATED: Consultancy: Biogen Canada, Novartis Canada, Genyzme Canada, Teva Neurosciences, Serono Canada, Comments: advisory board member; Payment for Manuscript Preparation: Biogen Canada, Comments: publication of review article; Travel/Accommodations/Meeting Expenses Unrelated to Activities Listed: Biogen Canada, Novartis Canada, Comments: travel to conferences and advisory boards; OTHER: Biogen Canada, ${ }^{*}$ Novartis Canada, ${ }^{*}$ Teva Neurosciences, ${ }^{*}$ Comments: clinical trial funding. Timothy J. Carroll—RELATED: Grant: National Institutes of Health,* Comments: I hold a National Institutes of Health research grant related to cerebral perfusion; UNRELATED: Grants/Grants Pending: National Institutes of Health, ${ }^{*}$ American Hospital Association, ${ }^{*}$ Comments: In addition to the National Institutes of Health and American Hospital Association grants that support this work, I have research grants that are independent of the work presented in this article. Richard I. Aviv—RELATED: Grant: Canadian Institutes of Health Research, ${ }^{*}$ Biogen. * *Money paid to the institution.

\section{REFERENCES}

1. Calabrese M, De Stefano N, Atzori M, et al. Detection of cortical inflammatory lesions by double inversion recovery magnetic resonance imaging in patients with multiple sclerosis. Arch Neurol 2007; 64:1416-22 CrossRef Medline

2. Calabrese M, Rocca MA, Atzori M, et al. A 3-year magnetic resonance imaging study of cortical lesions in relapse-onset multiple sclerosis. Ann Neurol 2010;67:376-83 CrossRef Medline

3. Deloire MS, Salort E, Bonnet M, et al. Cognitive impairment as marker of diffuse brain abnormalities in early relapsing remitting multiple sclerosis. J Neurol Neurosurg Psychiatry 2005;76:519-26 CrossRef Medline

4. Heaton RK, Nelson LM, Thompson DS, et al. Neuropsychological findings in relapsing-remitting and chronic-progressive multiple sclerosis. J Consult Clin Psychol 1985;53:103-10 CrossRef Medline

5. Calabrese M, Agosta F, Rinaldi F, et al. Cortical lesions and atrophy associated with cognitive impairment in relapsing-remitting multiple sclerosis. Arch Neurol 2009;66:1144-50 Medline

6. Rao SM, Leo GJ, Bernardin L, et al. Cognitive dysfunction in multiple sclerosis, I: frequency, patterns, and prediction. Neurology 1991; 41:685-91 CrossRef Medline

7. Calabrese M, Gallo P. Magnetic resonance evidence of cortical onset of multiple sclerosis. Mult Scler 2009;15:933-41 CrossRef Medline

8. Calabrese M, Poretto V, Favaretto A, et al. Cortical lesion load associates with progression of disability in multiple sclerosis. Brain 2012;135(pt 10):2952-61 CrossRef Medline

9. Seewann A, Enzinger C, Filippi M, et al. MRI characteristics of atypical idiopathic inflammatory demyelinating lesions of the brain: a review of reported findings. J Neurol 2008;255:1-10 Medline

10. Tardif CL, Bedell BJ, Eskildsen SF, et al. Quantitative magnetic resonance imaging of cortical multiple sclerosis pathology. Mult Scler Int 2012;2012:742018 CrossRef Medline

11. Chen JT, Easley K, Schneider C, et al. Clinically feasible MTR is sensitive to cortical demyelination in MS. Neurology 2013;80:246-52 CrossRef Medline

12. Poonawalla AH, Hasan KM, Gupta RK, et al. Diffusion-tensor MR imaging of cortical lesions in multiple sclerosis: initial findings. $R a$ diology 2008;246:880 - 86 CrossRef Medline

13. Calabrese M, Rinaldi F, Seppi D, et al. Cortical diffusion-tensor imaging abnormalities in multiple sclerosis: a 3-year longitudinal study. Radiology 2011;261:891-98 CrossRef Medline

14. Shin W, Horowitz S, Ragin A, et al. Quantitative cerebral perfusion using dynamic susceptibility contrast MRI: evaluation of reproducibility and age- and gender-dependence with fully automatic image postprocessing algorithm. Magn Reson Med 2007;58:1232-41 CrossRef Medline

15. Vakil P, Lee JJ, Mouannes-Srour JJ, et al. Cerebrovascular occlusive disease: quantitative cerebral blood flow using dynamic susceptibility contrast MR imaging correlates with quantitative $\mathrm{H} 2$ [15O] PET. Radiology 2013;266:879-86 CrossRef Medline

16. Aviv RI, Francis PL, Tenenbein R, et al. Decreased frontal lobe gray matter perfusion in cognitively impaired patients with secondaryprogressive multiple sclerosis detected by the bookend technique. AJNR Am J Neuroradiol 2012;33:1779-85 CrossRef Medline

17. Rashid W, Parkes LM, Ingle GT, et al. Abnormalities of cerebral perfusion in multiple sclerosis. J Neurol Neurosurg Psychiatry 2004; 75:1288-93 CrossRef Medline

18. Debernard L, Melzer TR, Van Stockum S, et al. Reduced grey matter perfusion without volume loss in early relapsing-remitting multiple sclerosis. J Neurol Neurosurg Psychiatry 2014;85:544-51 CrossRef Medline

19. Francis PL, Jakubovic R, O'Connor P, et al. Robust perfusion deficits in cognitively impaired patients with secondary-progressive multiple sclerosis. AJNR Am J Neuroradiol 2013;34:62-67 CrossRef Medline

20. Hojjat SP CC, Vitorino R, Feinstein A, et al. Regional reduction in cortical blood flow among cognitively impaired adults with relapsing-remitting multiple sclerosis patients. Mult Scler 2016 Jan 11. [Epub ahead of print] Medline

21. Hojjat SP CC, Carroll TJ, Vitorino R, et al. Perfusion reduction in the absence of structural differences in cognitively impaired versus unimpaired RRMS patients. Mult Sclel 2016 Feb 4. [Epub ahead of print] Medline

22. Polman $\mathrm{CH}$, Reingold SC, Banwell B, et al. Diagnostic criteria for multiple sclerosis: 2010 revisions to the McDonald criteria. Ann Neurol 2011;69:292-302 CrossRef Medline

23. Kurtzke JF. Rating neurologic impairment in multiple sclerosis: an expanded disability status scale (EDSS). Neurology 1983;33:1444-52 CrossRef Medline

24. Benedict RH, Fischer JS, Archibald CJ, et al. Minimal neuropsychological assessment of MS patients: a consensus approach. $\mathrm{Clin} \mathrm{Neu}$ ropsychol 2002;16:381-97 CrossRef Medline

25. Benedict RH, Bruce JM, Dwyer MG, et al. Neocortical atrophy, third ventricular width, and cognitive dysfunction in multiple sclerosis. Arch Neurol 2006;63:1301-06 CrossRef Medline

26. Shah MK, Shin W, Parikh VS, et al. Quantitative cerebral MR perfusion imaging: preliminary results in stroke. J Magn Reson Imaging 2010;32:796-802 CrossRef Medline

27. Ashburner J, Friston KJ. Unified segmentation. Neuroimage 2005;26: 839-51 CrossRef Medline

28. Ashburner J. A fast diffeomorphic image registration algorithm. Neuroimage 2007;38:95-113 CrossRef Medline

29. Jenkinson M, Beckmann CF, Behrens TE, et al. FSL. Neuroimage 2012;62:782-90 CrossRef Medline

30. Riccitelli G, Rocca MA, Pagani E, et al. Mapping regional grey and white matter atrophy in relapsing-remitting multiple sclerosis. Mult Scler 2012;18:1027-37 CrossRef Medline

31. Ranganath C, Johnson MK, D'Esposito M. Prefrontal activity associated with working memory and episodic long-term memory. Neuropsychologia 2003;41:378-89 CrossRef Medline

32. Leung HC, Gore JC, Goldman-Rakic PS. Sustained mnemonic response in the human middle frontal gyrus during on-line storage of spatial memoranda. J Cogn Neurosci 2002;14:659-71 CrossRef Medline

33. Zhang JX, Leung HC, Johnson MK. Frontal activations associated with accessing and evaluating information in working memory: an fMRI study. Neuroimage 2003;20:1531-39 CrossRef Medline

34. Law AS, Trawley SL, Brown LA, et al. The impact of working memory load on task execution and online plan adjustment during multitasking in a virtual environment. Q J Exp Psychol (Hove) 2013;66: 1241-58 CrossRef Medline

35. Suss HM, Oberauer K, Wittmann WW, et al. Working-memory capacity explains reasoning ability - and a little bit more. Intelligence 2002;30:161-288 CrossRef 
36. Wiley J, Jarosz AF. Working memory capacity, attentional focus, and problem solving. Curr Dir Psychol Sci 2012;21:258-62 CrossRef

37. Haxby JV, Petit L, Ungerleider LG, et al. Distinguishing the functional roles of multiple regions in distributed neural systems for visual working memory. Neuroimage 2000;11(5 pt 1):380-91 CrossRef Medline

38. Parry AM, Scott RB, Palace J, et al. Potentially adaptive functional changes in cognitive processing for patients with multiple sclerosis and their acute modulation by rivastigmine. Brain 2003;126(pt 12): 2750-60 CrossRef Medline

39. Köhler S, Black SE, Sinden M, et al. Memory impairments associated with hippocampal versus parahippocampal-gyrus atrophy: an MR volumetry study in Alzheimer's disease. Neuropsychologia 1998;36: 901-14 CrossRef Medline

40. Takashima A, Nieuwenhuis IL, Rijpkema M, et al. Memory trace stabilization leads to large-scale changes in the retrieval network: a functional MRI study on associative memory. Learn Mem 2007;14: 472-79 CrossRef Medline

41. Dutta R, McDonough J, Yin X, et al. Mitochondrial dysfunction as a cause of axonal degeneration in multiple sclerosis patients. Ann Neurol 2006;59:478-89 CrossRef Medline

42. Nikić I, Merkler D, Sorbara C, et al. A reversible form of axon damage in experimental autoimmune encephalomyelitis and multiple sclerosis. Nat Med 2011;17:495-99 CrossRef Medline

43. D'Haeseleer M, Hostenbach S, Peeters I, et al. Cerebral hypoperfusion: a new pathophysiologic concept in multiple sclerosis? J Cereb Blood Flow Metab 2015;35:1406-10 CrossRef Medline
44. De Keyser J, Steen C, Mostert JP, et al. Hypoperfusion of the cerebral white matter in multiple sclerosis: possible mechanisms and pathophysiological significance. J Cereb Blood Flow Metab 2008;28: 1645-51 CrossRef Medline

45. Ge Y, Zohrabian VM, Osa EO, et al. Diminished visibility of cerebral venous vasculature in multiple sclerosis by susceptibility-weighted imaging at 3.0 Tesla. J Magn Reson Imaging 2009;29:1190-94 CrossRef Medline

46. Tanaka R, Iwasaki $\mathrm{Y}$, Koprowski $\mathrm{H}$. Ultrastructural studies of perivascular cuffing cells in multiple sclerosis brain. Am J Pathol 1975;81:467-78 Medline

47. Adams CW, Poston RN, Buk SJ, et al. Inflammatory vasculitis in multiple sclerosis. J Neurol Sci 1985;69:269-83 CrossRef Medline

48. Woodroofe MN, Cuzner ML. Cytokine mRNA expression in inflammatory multiple sclerosis lesions: detection by non-radioactive in situ hybridization. Cytokin 1993;5:583-88 Medline

49. Sibson NR, Blamire AM, Perry VH, et al. TNF-alpha reduces cerebral blood volume and disrupts tissue homeostasis via an endothelinand TNFR2-dependent pathway. Brain 2002;125(pt 11):2446-59 CrossRef Medline

50. Fisk JD, Pontefract A, Ritvo PG, et al. The impact of fatigue on patients with multiple sclerosis. Can J Neurol Sci 1994;21:9-14 CrossRef Medline

51. Krupp LB, Elkins LE. Fatigue and declines in cognitive functioning in multiple sclerosis. Neurology 2000;55:934-39 CrossRef Medline 